disease $(n=53)$ reported moderate limitation for mobility. Patients with cardiovascular diseases reported moderate difficulties in carrying out daily activities.

* The values of each of the dimensions of the instrument are expressed in\%

** HBP: high blood pressure, CVD: cardiovascular disease, TB: tuberculosis

*** Mental disorders: anxiety, nervousness or depression.

Patients with rheumatic diseases $(n=2274)$ manifested different degrees of limitation in mobility and daily activities. Patients with rheumatic diseases and comorbidities had greater compromise in quality of life predominately by pain and physical discomfort (more than $50 \%$ of the patients) figure 1.

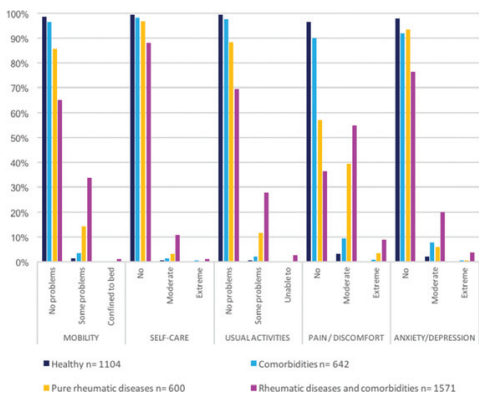

Abstract AB1244 - Figure 1. EQ5D-3L in patients with comorbidities, rheumatic diseases and healthy patients

Conclusions: In comparison with general population, rheumatic patients had a lower quality of life, and it is even worse in patients with rheumatic diseases and comorbidities. Comprehensive care of rheumatic patients should include strategies to improve standards of quality of life such as mobility, to perform daily activities and management of problems such as pain and discomfort. There are specific factors of intervention to reduce long-term disability of patients with rheumatic diseases.

Disclosure of Interest: None declared

DOI: 10.1136/annrheumdis-2018-eular.4319

\section{AB1245 $\quad$ EFFECTS OF A WORKPLACE-CENTRED COUNSELLING OF INDIVIDUALS WITH MUSCULOSKELETAL COMPLAINTS: A PROSPECTIVE COHORT STUDY}

H. Leiss, M. Hucke, M. Bécède, J. Smolen, K. Machold. Department of Rheumatology, Medical University of Vienna, Vienna, Austria

Background: Actively employed people with musculoskeletal complaints frequently seek medical advice only when symptoms have become chronic and have led to loss of workability.

Objectives: In this study, a brief examination was offered in the workplace setting in order to detect and to counsel individuals with symptoms of Rheumatic and musculoskeletal diseases(RMDs).

Methods: Employees of four companies were sent a screening questionnaire regarding musculoskeletal problems. In case of a positive screening, consultation by RMD specialists was offered which took place close to the workplace. If necessary, participants were referred to a practice/clinic specialised in RMDs (Orthopaedics, Rheumatology, Physical Medicine). Employees' work was categorised into physically highly demanding(HD) and less demanding(LD).

From participants consenting to follow-up, additional data were acquired: demographics, known pre-existing RMD, pain intensity, affected region(s), current treatment, number of sick leave-days due to musculoskeletal complaints, and out of pocket costs for treatments during the preceding year. General wellbeing and depression were measured by Euroquol-5d(EQ-5d) and Hospital Anxiety and Depression Scale(HADS). After one year, information about general wellbeing, pain intensity, treatment, individual costs, and days of sick leave during the intervening year was collected by telephone-interview.

Results: 6170 employees were invited.413 participated in the counselling program, 344 were enrolled in the study. $56.6 \%$ of the participants had no previously diagnosed RMD, after the specialists' assessment, this percentage decreased to $35.7 \%$. Men with LD workload had significantly higher wellbeing(EQ-5d scale):77.3 \pm 15.1 compared to women with both $\operatorname{LD}(71.0 \pm 20.1, p=0.034)$ and $H D$ $(64.6 \pm 21.3, p=0.001) . L D$ and HD differed significantly regarding percentage with painful upper(28.6 vs. $45.3, p=0.006)$ and lower(49.6 vs. $65.3, p=0.016)$ limbs.
Back pain was distributed equally among all groups. HD women reported significantly higher use of NSAIDs $(55.1 \%$ vs. $27.7 \%$ in female LD, $21.7 \%$ in male $\mathrm{HD}, 23.5 \%$ in male LD, $\mathrm{p}=0.001)$. HD men showed the lowest (4.1 \pm 2.9$)$, HD women showed the highest HADS anxiety-score $(6.3 \pm 3.8, \mathrm{p}=0.042)$.

235 individuals participated in telephone follow-up. There was significant improve ment in wellbeing(mean $77.2 \pm 17.4$ vs. $73.6 \pm 18.2$ at baseline, $p=0.006$ ) and in rating of RMD pain(mean $27.8 \pm 24.9$ vs. $40.8 \pm 24.6$ at baseline, $p=0.001$ ) Participants who were suspected by the specialist to suffer from RMDs had significantly increased out of pocket costs after one year(mean in $€ 441.8 \pm 861.6$ vs. $254.1 \pm 407.0, p=0.026)$. Use of NSAIDs decreased significantly from $29.1 \%$ to $17.4 \%, p=0.02$. Converesely, rates of use of physiotherapy (7.6 vs. $24.7, p=0.001$ ), gymnastics(2.7 vs. 23.4, $\mathrm{p}=0.001)$, physical therapy(12.8 vs. 43.3, $\mathrm{p}=0.027)$ and complementary/alternative methods $(7.4$ vs. $13.2, p=0.003)$ were significantly increased.

Conclusions: In our study we found most physical and psychological problems related to RMDs in HD working women. After one year, participants reported improved quality of life, reduction of RMD pain, higher utilisation of medical services and of gymnastics, less use of NSAIDs, and, if suspected to suffer from RMDs, higher out of pocket costs. Thus, this brief workplace-centred intervention appears to have had beneficial effects on both subjective well-being and on objective parameters suggesting improvements in physical/physiological health.

Disclosure of Interest: None declared

DOI: 10.1136/annrheumdis-2018-eular.4933

\section{AB1246 PROVISION OF RHEUMATOLOGY SERVICES TO 30 MILLION PEOPLE IN NORTH-WESTERN PAKISTAN (A NAÏVE DEPARTMENT WITH HUGE CHALLENGES)}

S. Khan ${ }^{1}$, I. Malik ${ }^{1}$, Z. Afridi ${ }^{2} .{ }^{1}$ Rheumatology; ${ }^{2}$ Pediatrics, Lady Reading Hospital, Medical Teaching Institution, Peshawar, Pakistan

Background: Practicing rheumatology needs multidisciplinary team work and also good funding. This becomes difficult in places where proper structure of healthcare is lacking.

Objectives: To share experience of establishing a Rheumatology unit in Khyber Pukhtunkhwa (KP) Pakistan.

Methods: KP is the Northwestern province of Pakistan. The population of KP is 30 million. The per capita income of Pakistan was 1180 US \$ in 2016.

Modern day treatments are expensive in rheumatology. Difficulties are in areas of expertise and biologics.

The first ever Rheumatology unit was established in Lady Reading Hospital (LRH) Peshawar in July 2017 which started its regular outpatient services. Problems at the start were absence of specialist nurses, junior doctors, special immunology and MSK Radiologists. Regular MSK ultrasound was started along with routine procedures. This had an enormous impact on the quality of care. Lack of awareness about rheumatic diseases in general population has been an issue which was addressed through newspapers, television and social media. The response was excellent. The outpatient numbers have raised, referral pathway was established and more patients are now seen in outpatients. Team was further built up by acquiring a trainee registrars and a consultant rheumatologist. Another problem was lack of proper patients education system due to lake of specialist nurses and non- availability of literature in local languages. Biologics are costly and very few people can afford these. Pakistan Bailtul Maal, a charitable organisation is the only way to provide biologics to patients on need basis. Currently only few biolog ics are available in the market i.e Etanercept, Rituximab and Tocilizumab. Adalimumab will come to market sometime in 2018.

Kids with Juvenile Idiopathic arthritis, Systemic Lupus Erythematosis and othe rheumatic problems are difficult to manage as there is no Paediatric Rheumatologist available in the entire province. We now have established a rapport with our paediatric colleagues which is working well.

Pakistan has only seven hospitals where training is offered in rheumatology but all are based in other provinces and none in KP. For this purpose we are in the process of establishing a dedicated rheumatology department where full training will be given to trainees according to international standards.

The data on rheumatic diseases is non-existing so we are now working on data collection on our local population.

Results: Working as a rheumatologist is a big challenge in under resourced areas. We have been having worst case scenario in almost all aspects. Howeve someone has to be at the forefront as millions of people have rheumatic diseases and they cannot be left alone with these conditions untreated. 


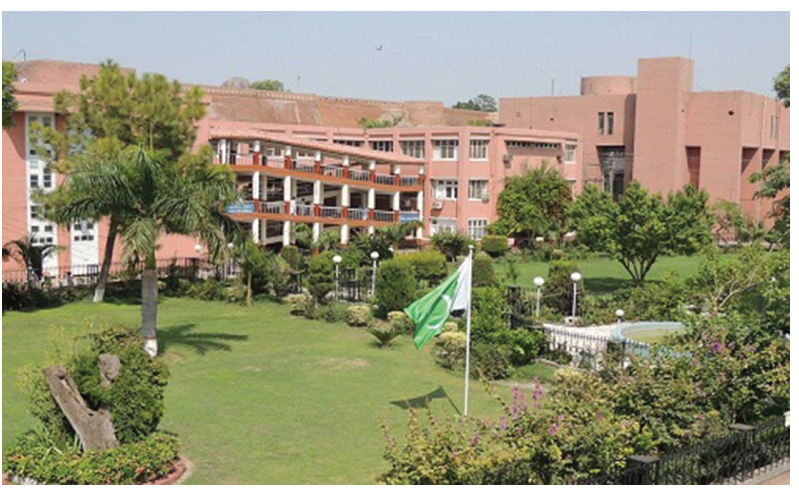

Abstract AB1246 - Figure 1

Conclusions: The idea is to persevere and continue efforts for the betterment of our patients. More specialists are needed to fill in the gaps along with appropriate funding to develop rheumatology services in our part of the country. We feel that situation in other countries with low socio-economics will be more or less the same or even worst for patients with rheumatic diseases. International communities and leagues such EULAR, BSR, ACR and APLAR etc should discuss this on their forums to see if in anyway they can improve lives of millions of people with rheumatic disorders in under-resourced countries.

Disclosure of Interest: None declared

DOI: 10.1136/annrheumdis-2018-eular.2297

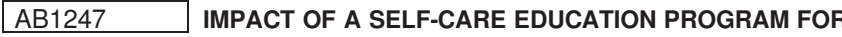 PATIENTS WITH OSTEOARTHRITIS}

A. Gurt ${ }^{1}$, J. Llorente ${ }^{2}$, M. López ${ }^{2}$, L. Tio ${ }^{2}$, B. Sena ${ }^{1}$, F. Montañes ${ }^{1}$, E. Marco ${ }^{3}$ J. Verges ${ }^{4}$, M. Herrero ${ }^{5}$, J. Monfort ${ }^{2}{ }^{1}$ CAP Vila Olimpica; ${ }^{2}$ Rheumatology; ${ }^{3}$ Rehabilitation, Hospital del Mar, ${ }^{4}$ OAFI foundation; ${ }^{5}$ Bioiberica S.A, Barcelona, Spain

Background: Osteoarthritis (OA) has a prevalence rate that reaches $29 \%$ in people older than 60 years ${ }^{1}$. Treatments available are limited. The costs of OA in Spain are about 4.800 million euros/year ${ }^{2}$.

Objectives: To create a self-care program for $\mathrm{OA}$ patients in order to improve their quality of life $(\mathrm{Q} o \mathrm{~L})$ and therefore to reduce the socioeconomic cost.

Methods: The design of the program was carried out by 2 PC physicians, 1 rheumatologist, 1 rehabilitator, 1 nurse and 2 psychologists. The program included a total of 9 sessions of 1.5 hours once a week. There were 2 sessions for each of the following topics: general information, physical activity, nutrition, coping with the disease, and 1 summary session. Three OA patients were trained and afterwards they were in charge of imparting the program to other patients with the assistance of a nurse. Before and after the program some data was collected relating the patients' knowledge, food and physical activity habits, social networks and hours of rest. They were asked to complete WOMAC, EuroQol-5D, and HAD Scale questionnaires. The statistical analysis was performed using package SPSS v16.

Results: 60 Knee OA patients were recruited from Hospital del Mar and Vila Olímpica PC centre, and divided into 6 different groups. Only the results of the first two groups are shown. Group1 (11 patients) and 2 (10 patients). First we analysed differences between the basal data and the ones collected after the last session. The analysis of the data from all the patients (groups 1 and 2) showed that in knowledge about OA management the average value obtained in the basal visit was $6.31 \pm 2.798$, and $7.81 \pm 1.94$ after the last session $(p=0.024)$. Analysis of pain by VAS showed that the average value in the basal visit was $3.91 \pm 1.82$, and 2.44 \pm 2.03 after the last session $(p=0.014)$. We observe a tendency, although it doesn't reach significant differences in QoL, where the average value in the basal visit was $2.31 \pm 1.81$, and $1.63 \pm 1.54$ after the last session $(p=0.052)$. In the HADS scale the average value in the basal visit was $9.86 \pm 6.02$, and $8.36 \pm 5.40$ after the last session ( $p=0.052)$. Regarding the analysis of differences between two groups, categorical items were analysed. In the total questionnaire at the end of the sessions the average for group 1 was 6.89 and for group 2 was $8.75(p=0.038)$. Meeting with friends frequency at the beginning and at the end of the study was also different among groups $(p=0.014 / p=0.019)$.

Conclusions: This self-care education program had a positive effect on the $\mathrm{OA}$ patients pain perception, and it could also be observed an improvement in QoL and the anxiety and depression. As differences among groups were also observed, socioeconomic and education aspects must be considered in the future.
REFERENCES:

[1] Fernandez-Lopez JC, et al. EPISER StudyGroup. Prevalence, risk factors, and impact of knee pain suggesting osteoarthritis in Spain. Clin Exp Rheumatol. 2008;26(2):324-32.

[2] Loza E, Lopez-Gomez JM, et al. Economic burden of knee and hip osteoarthritis in Spain. Arthritis Rheum. 2009 15;61(2):158-65.

Disclosure of Interest: None declared

DOI: 10.1136/annrheumdis-2018-eular.6837

\section{AB1248 VACCINATION RATE IN PATIENTS WITH RHEUMATIC DISEASES: A CROSS-SECTIONAL STUDY IN MEXICAN PATIENTS}

J.P. Carrizales-Luna, D. Galarza-Delgado, J. Esquivel-Valerio, G. Serna-Peña, L. Ramírez-Monterrubio, L. Lozano-Plata, I. Hernández-Galarza, T. GraciaArechiga. Rheumatology, Universidad Autónoma de Nuevo León, Monterrey, Mexico

Background: Patients with rheumatic diseases have an increased risk of infections, which can be prevented with vaccination schemes. The vaccination rate in rheumatic patients is low in Mexico (17\%-25\%), mainly because of the lack of physician recommendation. Vaccination strategies have been shown to improve adherence to the application.

Objectives: The objectives of the study was to determine the vaccination rate in patients with rheumatic diseases and the main reasons of failed vaccination. Methods: We questioned 84 patients from the rheumatology clinic of the Univesitary Hospital "José E. González", Mexico, from June to July 2017. The vaccination status and the reasons for failed vaccination were recorded.

Results: The majority of patients were women (89.3\%), average age 50 years (17-81 years). Most of the patients had rheumatoid arthritis (45.3\%), followed by other autoimmune diseases (27.4\%) and non-autoimmune diseases (27.4\%). The highest rates of vaccination were for tetanus-diphtheria (44\%), influenza (39.3\%) and pneumococcus $(31 \%)$. They were lower for hepatitis B virus $(9.8 \%)$, human papilloma virus (4.8\%), hepatitis A virus (2.4\%) and herpes zoster virus ( $0 \%)$. The main reason for failed vaccination was the lack of indication from the physician $(34.5 \%)$. If indicated, $89.3 \%$ of the patients would accept to be vaccinated

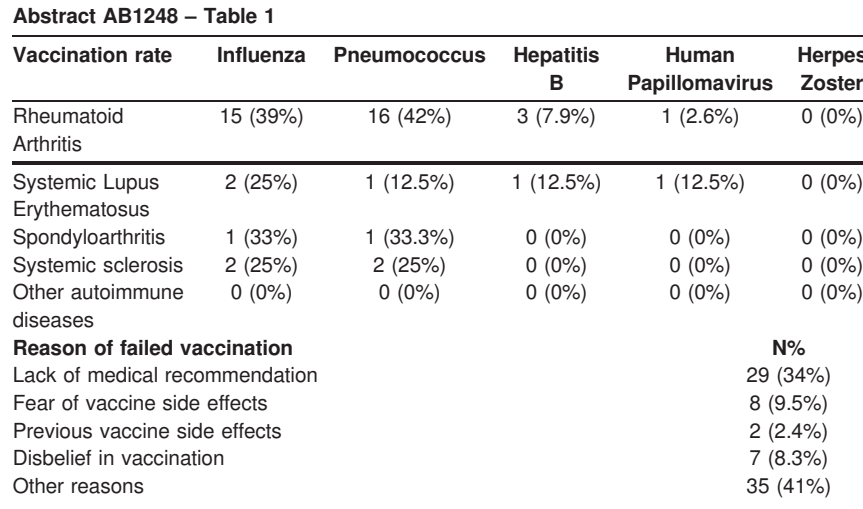

Conclusions: We found a low vaccination rate in the rheumatology clinic. We found an insufficient promotion and indication of vaccination by the rheumatology staff. Constant fomentation and updated knowledge about vaccination recom mendations in autoinmune diseases is necessary for the prevention of infections and to improve the comprehensive care of patients with rheumatic diseases.

\section{REFERENCES}

[1] Garg S. ACR Abstracts. 2016.

[2] Kostantinos T \& Vassilopoulus D. Best Practice \& Research Clinical Rheumatology xxx (2016) 1e18.

[3] Black S, et al. ACR Abstracts. 2011.

[4] Bühler S, et al. Swiss Med Wkly. 2015;145: w14159

Disclosure of Interest: None declared

DOI: 10.1136/annrheumdis-2018-eular.6092 